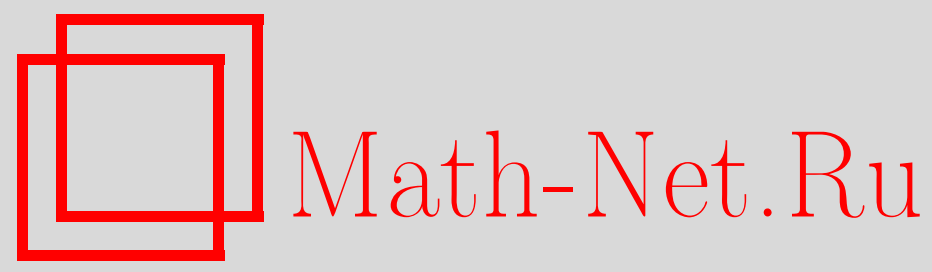

А. А. Туганбаев, Характеристические подмодули инъективных модулей, Дискрет. матем., 2013, том 25, выпуск 2, 8590

DOI: https://doi.org/10.4213/dm1237

Использование Общероссийского математического портала Math-Net.Ru подразумевает, что вы прочитали и согласны с пользовательским соглашением http://www.mathnet.ru/rus/agreement

Параметры загрузки:

IP : 3.85 .7 .115

26 апреля 2023 г., 15:10:59

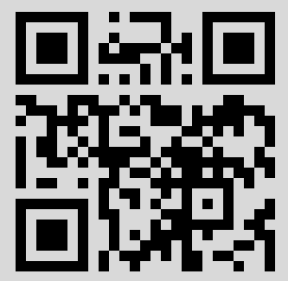


УДК 512.553.8

DOI $10.4213 / \mathrm{dm} 1237$

\title{
Характеристические подмодули инъективных модулей
}

\author{
() 2013 г. А. А. Туганбаев
}

\begin{abstract}
Исследуются модули, являющиеся характеристическими подмодулями своих инъективных оболочек.

Автор поддержан Российским фондом фундаментальных исследований. Проект 08-01-00693-а: Структурная теория колец.
\end{abstract}

Все кольца в этой статье предполагаются ассоциативными и с ненулевой единицей, а модули унитарными. Модуль называется нетеровым, если каждая строго возрастающая цепь его подмодулей конечна. Слова типа " $A$ - нетерово кольцо" означают, что $A_{A}$ и ${ }_{A} A-$ нетеровы модули. Модуль $M$ называется инбективным относительно модуля $X$ или $X$-ингективным, если для любого подмодуля $X_{1}$ в $X$ каждый гомоморфизм $X_{1} \rightarrow M$ продолжается до гомоморфизма $X \rightarrow M$. Модуль $M$ над кольцом $A$ называется инбективным, если $M$ инъективен относительно любого $A$-модуля. Подмодуль $M$ модуля $E$ называется существенным, если $M \cap E_{1} \neq 0$ для любого ненулевого подмодуля $E_{1}$ в $E$. Если $E$ - инъективный модуль и $M-$ существенный подмодуль в $E$, то $E$ называется инбективной оболочкой модуля $M$. Инъективная оболочка единственна с точностью до изоморфизма. Подмодуль $M$ модуля $E$ называется характеристическим, если $f(M) \subseteq M$ для каждого автоморфизма $f$ модуля $E$.

В работах [3], [8], [10] модуль называется автоморфизм-инвариантным, если он является характеристическим подмодулем своей инъективной оболочки. Нетрудно проверить, что модуль $M$ является автоморфизм-инвариантным в точности тогда, когда $M$ - характеристический подмодуль некоторого инъективного модуля; см. замечание 6 ниже.

Замечание 1. В [3, Theorem 16] доказано, что модуль $M$ является автоморфизминвариантным модулем в точности тогда, когда $M$ - псевдоинбективный модуль, т.е. для любого подмодуля $X$ в $M$ каждый мономорфизм $X \rightarrow M$ продолжается до эндоморфизма модуля $M$. О псевдоинъективных модулях см., например, [6], [11], $[3]$.

Замечание 2. Ясно, что каждый инъективный модуль является автоморфизминвариантным. Обратное неверно, поскольку каждая простая абелева группа является конечным автоморфизм-инвариантным неинъективным модулем над кольцом целых чисел $\mathbb{Z}$.

Кольцо $A$ называется правым колъцом Голди, если $A$ не содержит бесконечных прямых сумм ненулевых правых идеалов и $A$ - кольцо с условием максимальности 
для правых аннуляторов. Кольцо называется первичным, если произведение любых двух его ненулевых идеалов не равно нулю. Пусть $M$ - правый модуль над кольцом $A$ и $t(M)$ - множество всех элементов из $M$, аннулируемых некоторыми неделителями нуля кольца $A$. Модуль $M$ называется непериодическим (соответственно, периодическим, модулем без кручения), если $t(M) \neq M$ (соответственно, $t(M)=M$, $t(M)=0)$.

В [6, Theorem 6] доказано, что каждый псевдоинъективный модуль без кручения над первичным правым кольцом Голди инъективен. В связи с этим и замечанием 1 мы докажем теорему 1, которая является первым основным результатом работы.

Теорема 1. Пусть $A$ - первичное правое кольцо Голди и $M$ - непериодический правый А-модуль. Равносильны условия:

1) $M-$ автоморфизм-инвариантный модуль;

2) $M-$ ингективный модуль.

Модуль называется квазиингективным или самоинбективным, если он инъективен относительно себя.

Замечание 3. Хорошо известно, что модуль $M$ квазиинъективен в точности тогда, когда $f(M) \subseteq M$ для любого эндоморфизма $f$ инъективной оболочки модуля $M$, см. [7] или [13, п. 17.11]. Ясно, что каждый квазиинъективный модуль является автоморфизм-инвариантным (псевдоинъективным). Пусть $\left\{F_{i=1}^{\infty}\right\}$ - счетное множество экземпляров поля $\mathbb{Z} / 2 \mathbb{Z}$ и $A$ - подкольцо прямого произведения всех $F_{i}$, состоящее из всех последовательностей, стабилизирующихся на конечном шаге. В [3, Example 9] показано, что $A$ - автоморфизм-инвариантный модуль, не являющийся квазиинъективным.

Модуль называется наследственным, если все его подмодули проективны. Кольцо $A$ называется ограниченным, если каждый его существенный правый или левый идеал содержит ненулевой идеал. Заметим, (см. [9]), что каждое наследственное нетерово первичное кольцо или ограничено, или примитивно справа и слева.

Замечание 4. В [6, теорема 5] доказано, что каждый псевдоинъективный периодический модуль над ограниченным наследственным нетеровым первичным кольцом квазиинъективен.

В связи с замечанием 4 и с замечанием 3 мы докажем теорему 2 , которая является вторым основным результатом данной работы.

Теорема 2. Пусть $A$ - ограниченное наследственное нетерово кольио. Для $A$ модуля М равносильны условия:

1) $М$ - автоморфизм-инвариантный модуль;

2) $M$ - либо квазиингективный периодический модуль, либо ингективный непериодический модуль.

Доказательство теорем 1 и 2 разбито на ряд утверждений, некоторые из них представляют самостоятельный интерес.

Пусть $A$ - кольцо и $M-$ правый $A$-модуль. Через $\operatorname{Sing}(M)$ обозначается подмодуль в $M$, состоящий из всех элементов модуля $M$, аннуляторы которых в $A$ являются существенными правыми идеалами. Модуль $M$ называется несингулярным (соответственно, сингулярным), если $\operatorname{Sing}(M)=0($ соответственно, $\operatorname{Sing}(M)=M)$. 
Если $X$ - существенный подмодуль модуля $M$, то $M$ называется существенным расширением модуля $X$. Подмодуль $X$ модуля $M$ называется замкнутым в $M$, если $X=X^{\prime}$ для каждого подмодуля $X^{\prime}$ в $M$, являющегося существенным расширением модуля $X$. Модуль называется равномерным, если любые два его ненулевых подмодуля имеют ненулевое пересечение. Другие используемые в работе определения содержатся, например, в [13] и [12].

Модуль $M$ называется автоморфизм-продолжаемым, если для любого подмодуля $X$ в $M$ каждый автоморфизм модуля $X$ продолжается до эндоморфизма $M$.

Замечание 5. Из замечания 1 следует, что каждый автоморфизм-инвариантный (т.е. псевдоинъективный) модуль является автоморфизм-продолжаемым. Однако не все автоморфизм-продолжаемые модули автоморфизм-инвариантны. Например, кольцо целых чисел $\mathbb{Z}$ является автоморфизм-продолжаемым $\mathbb{Z}$-модулем, поскольку произвольный автоморфизм любого ненулевого подмодуля в $\mathbb{Z}_{\mathbb{Z}}$ есть умножение на 1 или -1 . При этом модуль $\mathbb{Z}_{\mathbb{Z}}$ не является автоморфизм-инвариантным, поскольку его инъективная оболочка $\mathbb{Q}_{\mathbb{Z}}$ имеет такой автоморфизм $\varphi: q \rightarrow q / 2$, что $\varphi(\mathbb{Z}) \nsubseteq \mathbb{Z}$.

Замечание 6. Для модуля $M$ равносильны условия:

1) $M$ - автоморфизм-инвариантный модуль;

2) $M$ - характеристический подмодуль некоторого инъективного модуля $Q$.

В замечании 6 импликация 1) $\Rightarrow 2$ ) очевидна. Импликация 2) $\Rightarrow 1$ ) следует из того, что $Q=X \oplus Y$, где $X$ - инъективная оболочка модуля $M$, и каждый автоморфизм $\alpha$ модуля $X$ продолжается до автоморфизма $\beta$ модуля $Q$ с помощью правила $\beta(x+y)=$ $\alpha(x)+y$.

Лемма 1. Пусть $M-$ модуль и $X, Y-$ подмодули в $M$ с условием $X \cap Y=0$. Если $f: Y \rightarrow X$ - гомоморфизм, то существует такой автоморфизм а модуля $X \oplus Y$, что эндоморфизм $\alpha-1$ модуля $X \oplus Y$ совпадает с $f$ на $Y$.

Доказательство. Определим эндоморфизм $\alpha$ модуля $Z$ соотношением $\alpha(x+y)=$ $x+f(y)+y$ для всех $x \in X$ и $y \in Y$. Допустим, что

$$
0=\alpha(x+y)=x+f(y)+y, \quad x \in X, y \in Y .
$$

Тогда $\alpha-$ мономорфизм, поскольку

$$
y=-x-f(y) \in X \cap Y=0, \quad f(y)=0, \quad x=x+f(y)+y=\alpha(x+y)=0 .
$$

Кроме того, для любых $x \in X$ и $y \in Y$

$$
x+y=(x-f(y))+(f(y)+y)=\alpha(x-f(y))+\alpha(y) \in \alpha(X \oplus Y) .
$$

Поэтому $\alpha$ - искомый автоморфизм модуля $X \oplus Y$.

Лемма 2. Пусть $A$ - кольцо, $M$ - автоморфизм-продолжсаемый правый $A$-модуль и $X, Y-$ подмодули в $M$ с условием $X \cap Y=0$.

1) Если $f: Y \rightarrow X-$ гомоморфизм, то существует эндоморфизм $g$ модуля $M$, который совпадает с $f: Y \rightarrow X$ на $Y$.

2) Если $M=X \oplus Y$, то модуль $X$ ингективен относительно $Y$.

3) Если модуль $M / X$ несингулярен, то модуль $X$ инбективен относительно $Y$. 
Доказательство. 1) По лемме 1 существует такой автоморфизм $\alpha$ модуля $X \oplus Y$, что эндоморфизм $\alpha-1_{X \oplus Y}$ модуля $X \oplus Y$ совпадает с гомоморфизмом $f: Y \rightarrow X$ на $Y$. Так как $M$ - автоморфизм-продолжаемый модуль, то автоморфизм $\alpha$ модуля $X \oplus Y$ продолжается до эндоморфизма $\beta$ модуля $M$. Обозначим через $g$ эндоморфизм $\beta-1_{M}$ модуля $M$. Тогда $g$ совпадает с $f$ на $Y$.

2) Пусть $Y_{1}$ - подмодуль в $Y$ и $f_{1}$ - гомоморфизм из $Y_{1}$ в $X$. По 1$)$ существует эндоморфизм $g$ модуля $M$, который совпадает с $f_{1}: Y_{1} \rightarrow X$ на $Y_{1}$. Пусть $\pi-$ проекция модуля $M=X \oplus Y$ на $X$ с ядром $Y$ и $u: Y \rightarrow M$ - естественное вложение. Обозначим через $f$ гомоморфизм $\pi g u$ из $Y$ в $X$. Тогда $f$ совпадает с $f_{1}$ на $Y_{1}$. Поэтому модуль $X$ инъективен относительно $Y$.

3) Пусть $Y_{1}$ - подмодуль в $Y$ и $f_{1}$ - гомоморфизм из $Y_{1}$ в $X$. По лемме Цорна существует такой подмодуль $Z$ в $Y$, что $Y_{1} \cap Z=0$ и $Y_{1} \oplus Z$ - существенный подмодуль в $Y$. Обозначим $Y_{2}=Y_{1} \oplus Z$. Гомоморфизм $f_{1}: Y_{1} \rightarrow X$ продолжается до гомоморфизма $f_{2}: Y_{2} \rightarrow X$ с помощью соотношения $f_{2}\left(y_{1}+z\right)=f_{1}\left(y_{1}\right)$. По 1$)$ существует эндоморфизм $g$ модуля $M$, который совпадает с $f_{2}: Y_{2} \rightarrow X$ на $Y_{2}$.

Остается доказать, что $g(y) \in X$ для любого элемента $y$ модуля $Y$. Пусть $h: M \rightarrow$ $M / X$ - естественный эпиморфизм. Так как $Y_{2}$ - существенный подмодуль в $Y$, то $y B \subseteq Y_{2}$ для некоторого существенного правого идеала $B$ кольца $A$; см., например, $[12,4.4(1)]$. Тогда

$$
\begin{gathered}
g(y) B=g(y B) \subseteq g\left(Y_{2}\right)=f_{2}\left(Y_{2}\right)=f_{1}\left(Y_{1}\right) \subseteq X, \\
h(g(y)) B=h(g(y) B) \subseteq h(X)=0 .
\end{gathered}
$$

Поэтому $h(g(y)) \in \operatorname{Sing}(M / X)=0$ и $g(y) \in \operatorname{Ker}(h)=X$.

Для удобства мы соберем в леммах 3,4 и 5 несколько хорошо известных результатов.

Лемма 3. Пусть $A$ - кольцо, $n$ - натуральное число, $X$ и $Y$ - правые $A$-модули, причем $X$ является $Y$-инбективным.

1) $X-\bar{Y}$-ингективный модуль для любого подфактора $\bar{Y}$ модуля $Y^{n}$.

2) Каждый модуль, инбективный относительно модуля $A_{A}$, является инбективным модулем.

3) Если модуль $Y^{n}$ содержит изоморфную копию модуля $A_{A}$, то модуль $X$ ингективен.

Утверждения 1) и 2) леммы 3 содержатся, например, в [13, пп. 16.2 и 16.4], а утверждение 3) вытекает из 1) и 2).

Лемма 4. Пусть $A$ - полупервичное правое кольцо Голди и $M$ - правый $A$-модуль.

1) $\operatorname{Sing}(M)=t(M)$. В частности, $M$ несингулярен (соответственно, сингулярен) в точности тогда, когда $M$ - периодический модуль (соответственно, модуль без кручения).

2) Существенное расширение любого сингулярного правого $A$-модуля является сингулярным модулем.

3) Если существует такой подмодуль $X$ в $M$, что модули $X$ и $M / X$ сингулярны, то модуль $М$ сингулярен.

4) $\operatorname{Sing}(M)$ - замкнутый подмодуль в $M$ и модуль $M / \operatorname{Sing}(M)$ несингулярен. 
Доказательство. 1) Утверждение доказано в [5, Proposition 6.9].

2) и 3) Утверждения доказаны в [5, Proposition 6.10].

4) Утверждение следует из 1) и 2).

Лемма 5. Пусть $A$ - первичное правое кольцо Голди и $Y$ - ненулевой несингулярнъй правый $A$-модуль.

1) Существует такое натуральное число п, что модуль $Y^{n}$ содержит изоморфную копию модуля $A_{A}$.

2) Если $X-Y$-ингективный $A$-модуль, то $X$ - ингективный модуль.

В лемме 5 утверждение 1) доказано в [5, Corollary 6.25], а утверждение 2) вытекает из 1) и леммы $3(3)$.

Лемма 6. Пусть $A$ - первичное правое колъцо Голди и $M$ - несингулярный автоморфизм-продолжаемый правый $A$-модуль.

1) Если $X$ - собственный замкнутый подмодуль в $M$, то $X$ - ингективный модуль.

2) Если $M$ - неравномерный модуль, то $M-$ ингективный модуль.

Доказательство. 1) Так как $X$ - собственный замкнутый подмодуль несингулярного модуля $M$, то модуль $M / X$ несингулярен и $X \cap Y=0$ для некоторого ненулевого подмодуля $Y$ в $M$. По леммам 1 и 2(3) модуль $X$ инъективен относительно $Y$. По лемме 5(2) модуль $X$ инъективен.

2) Так как $M$ - несингулярный неравномерный модуль, то $M$ - существенное расширение модуля $X_{1} \oplus X_{2}$, где $X_{1}$ и $X_{2}$ - ненулевые несингулярные замкнутые подмодули в $Y$. По 1$)$ модули $X_{1}$ и $X_{2}$ инъективны. Тогда $X_{1} \oplus X_{2}$ - существенное инъективное прямое слагаемое модуля $M$. Поэтому $M=X_{1} \oplus X_{2}$ и $M$ инъективен.

Лемма 7. Пусть $A$ - первичное правое кольцо Голди, $M$ - автоморфизмпродолжаемый правый $A$-модуль и $M \neq \operatorname{Sing}(M)$.

1) $\operatorname{Sing}(M)$ - ингективный модуль.

2) Существует прямое разложение $M=\operatorname{Sing}(M) \oplus Y$, где $Y$ - ненулевой несингулярный автоморбизм-продолжаемый модуль, причем либо модуль $М$ инбективен и модуль $Y$ равномерен, либо $Y$ - автоморфизм-продолжаемый равномерный неинбективный модуль.

Доказательство. Обозначим $X=\operatorname{Sing}(M)$.

1) По лемме 4(4) $X$ - замкнутый подмодуль в $M$. Так как $X \neq M$, то по лемме 6(1) $X$ - инъективный модуль.

2) Так как $X$ - собственный инъективный подмодуль модуля $M$, то по 1) существует прямое разложение $M=X \oplus Y$, где $Y$ - ненулевой несингулярный автоморфизм-продолжаемый модуль. Если $Y$ - неравномерный модуль, то по лемме 6(2) $Y$ - инъективный модуль. Тогда $M=X \oplus Y$ - инъективный модуль.

Лемма 8 ([1, 3.5]). Если $M$ - прямая сумма равномерных модулей, то $M$ является квазиинбективным модулем в точности тогда, когда $M$ - псевдоинбективный модуль. 
Замечание 7 (Окончание доказательства теоремы 1). Импликация 2) $\Rightarrow$ 1) следует из того, что любой инъективный модуль является автоморфизм-инвариантным.

$1) \Rightarrow 2)$. Пусть $A$ - первичное правое кольцо Голди и $M$ - автоморфизминвариантный непериодический правый $A$-модуль. По лемме 4(4), лемме $7(2)$ и замечанию 1 можно считать, что $M=X \oplus Y$, где $X$ - инъективный сингулярный модуль, $Y$ - ненулевой несингулярный равномерный автоморфизм-инвариантный модуль. По лемме 8 модуль $Y$ - квазиинъективный. По лемме 5(2) $Y$ - инъективный модуль. Тогда $M=X \oplus Y-$ инъективный модуль.

Предложение 1. Пусть $A$ - ограниченное наследственное нетерово первичное кольио. Для $A$-модуля $M$ равносильны условия:

1) $М$ - автоморфизм-инвариантный модуль;

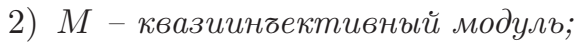

3) $M$ - либо квазиинбективный периодический модуль, либо инбективный непериодический модуль.

Доказательство. Импликация 1) $\Rightarrow 3$ ) вытекает из теоремы 1 и замечания 4.

Импликация 2) $\Rightarrow 1$ ) следует из замечания 3 .

Импликация 3) $\Rightarrow 2$ ) проверяется непосредственно.

Замечание 8. Теорема 2 вытекает из предложения 1.

\section{Список литературы}

1. Alahmadi A., Er N, Jain S. K., Modules which are invariant under monomorphisms of their injective hulls. J. Australian Math. Soc. (2005) 79, №3, 2265-2271.

2. Er N., Rings whose modules are direct sums of extending modules. Proc. Amer. Math. Soc. (2009) 137, №7, 2265-2271.

3. Er N., Singh S., Srivastava A.K.. J. Algebra (2013) 379, 223-229.

4. Goodearl K. R., Ring Theory: Nonsingular Rings and Modules. Marcel Dekker, New York, 1976.

5. Goodearl K. R., Warfield R. B., An Introduction to Noncommutative Noetherian Rings. Cambridge University Press, Cambridge, 1989.

6. Jain S. K., Singh S., Quasi-injective and pseudo-injective modules. Canadian Math. Bull. (1975) 18, №3, 359-366.

7. Johnson R. E., Wong F. T., Quasi-injective modules and irreducible rings. J. London Math. Soc. (1961) 36, 260-268.

8. Lee T. K., Zhou Y., Modules which are invariant under automorphisms of their injective hulls. J. Algebra Appl. (2013) 6, №2,.

9. Lenagan T. H., Bounded hereditary Noetherian prime rings. J. London Math. Soc. (1973) 6, 241-246.

10. Singh S., Srivastava A. K., Rings of invariant module type and automorphism-invariant modules Contemporary Mathematics (2013).

11. Teply M. L., Pseudo-injective modules which are not quasiinjective. Proc. Amer. Math. Soc. (1975) 49, №2, 305-310.

12. Tuganbaev A., Semidistributive Modules and Rings. Kluwer Academic Publishers, Dordrecht-Boston-London, 1998.

13. Wisbauer R., Foundations of Module and Ring Theory. Gordon and Breach, Philadelphia, 1991. 